\title{
Discordancia angiográfico-funcional posimplante valvular aórtico percutáneo. Reporte de caso
}

\author{
Angiographical-functional disorder post percutaneous aortic valve \\ replacement
}

Alicia Lorena Terragno', Leandro Gastón Puerta', Tomás Valverde², Gabriel Fernando Dionisio³

\section{RESUMEN}

El implante valvular aórtico percutáneo se ha vuelto una alternativa a la cirugía de reemplazo valvular en aquellos pacientes de alto riesgo quirúrgico. Pese a ser un procedimiento menos invasivo que la cirugía a cielo abierto, existen factores que aumentan la morbimortalidad de estos pacientes. Uno de ellos es la presencia de insuficiencia aórtica moderada a severa, por lo que su rápido diagnóstico y tratamiento es de suma importancia. Por este motivo, se han evaluado parámetros hemodinámicos que permitirían determinar el impacto de esta condición, siendo de utilidad para definir la conducta terapéutica destinada a corregirla.

Presentamos el caso de una paciente que, luego de someterse a un implante valvular aórtico percutáneo, en un aortograma control inmediato se diagnosticó insuficiencia aórtica severa. Al evaluarse el índice de regurgitación aórtico (IRA) pudo observarse que era $>$ a 25 , por lo que se asumió como funcionalmente irrelevante, y se dio por finalizado el procedimiento. Luego, durante la internación, se realizó un ecocardiograma control que evidenció insuficiencia valvular leve, con buena evolución clínica post implante, corroborando de esta manera los resultados arrojados por el IRA.

Palabras claves: estenosis aórtica severa, implante valvular aórtico percutáneo, leak paravalvular.

\begin{abstract}
The percutaneous aortic valve implant has become an alternative to valvular replacement surgery in those patients at high surgical risk. Despite being a less invasive procedure than open surgery, there are factors that increase the morbidity and mortality of these patients. One of them is the presence of moderate to severe aortic insufficiency, so its rapid diagnosis and treatment is of extreme importance. For this reason, hemodynamic parameters have been evaluated that would allow to determine the impact of this condition, being useful to define the therapeutic behavior destined to correct it.

We present the case of a patient who, after undergoing a percutaneous aortic valve implant, was diagnosed with severe aortic insufficiency in an immediate control aortogram. When the aortic regurgitation index (ARI) was evaluated, it could be observed that it was $>25$, so it was assumed to be functionally irrelevant, and the procedure was terminated. Then, during the hospitalization, a control echocardiogram was performed that showed mild valvular insufficiency, with good clinical evolution post implant, corroborating in this way the results thrown by the ARI.
\end{abstract}

Keywords: severe aortic stenosis, percutaneous aortic valve implantation, paravalvular leak.

\section{INTRODUCCIÓN}

El reemplazo valvular aórtico percutáneo (TAVI) ha demostrado no ser inferior a la cirugía de recambio valvular e inclusive mas beneficioso en aquellos pacientes de alto riesgo quirúrgico. No obstante, existen determinantes que modifican la morbimortalidad en estos individuos ${ }^{1,2}$.

Uno de ellos es la insuficiencia aórtica moderada a severa secundaria a leak paravalvular, cuya incidencia es aproximadamente del 15-20\%, siendo esta última la que genera mayor repercusión en términos de morbimortalidad ${ }^{1-3}$.

Contar con parámetros que nos permitan evaluar rápida y efectivamente la repercusión hemodinámica posimplante inmediato, de la insuficiencia aórtica significativa secundaria a leak perivalvular, puede permitir definir una conducta terapéutica correctiva, no únicamente en base al resultado angiográfico.

Presentamos el caso de una paciente que luego de realizarse un reemplazo valvular aórtico percutáneo, y presentar en el aortograma control posterior al procedimiento insuficiencia valvular moderada a severa, se procedió a calcular el índice de regurgitación aórtica (IRA) para definir la conducta terapéutica a seguir.

Médico de Staff. Servicio de Hemodinamia, Clínica Modelo Lanús.

2. Fellow de Hemodinamia. Servicio de Hemodinamia, Clínica Modelo Lanús

3. Jefe de Servicio. Servicio de Hemodinamia, Clínica Modelo Lanús.

$\triangle$ Correspondencia: alicia-terragno@hotmail.com

Los autores no declaran conflictos de intereses

Recibido:07/09/2018|Aceptado: 27/11/2018

\section{CASO CLÍNICO}

Paciente de sexo femenino de 84 años, que como factores de riesgo cardiovascular presenta hipercolesterolemia y, como antecedentes patológicos, estenosis aórtica severa sintomática por disnea en clase funcional II y cáncer de mama por el que recibió tratamiento con radio- y quimioterapia en el año 2009. Dado el cuadro clínico de la paciente y tras ser evaluada por el heart team, se decide proceder al implante valvular aórtico percutáneo por presentar elevada fragilidad (test de caminata en $5 \mathrm{mts}<0,5 \mathrm{mts} / \mathrm{s}$; índice de Katz 2 puntos; minimental test normal) y fibrosis actínica del tórax.

En el ecocardiograma transesofágico preimplante se observó estenosis aórtica severa, presentando área valvular de 0,5 $\mathrm{cm}^{2}$, velocidad pico $5,1 \mathrm{~m} / \mathrm{s}$ y gradiente transvalvular medio de $70 \mathrm{mmHg}$, función sistólica del ventrículo izquierdo conservada, cavidades cardíacas con diámetros conservados y sin alteraciones en la motilidad segmentaria. En el análisis tomográfico se evidenció un perímetro del anillo valvular aórtico de $73 \mathrm{~mm}$, con área de $398 \mathrm{~mm}^{2}$ y un diámetro valvular promedio de $25 \mathrm{~mm}$.

En la espirometría presentó restricción leve de la pequeña y mediana vía aérea. La cinecoronariografía no evidenció lesiones coronarias significativas y el electrocardiograma mostró ritmo sinusal asociado a bloqueo completo de rama derecha.

Se realizaron los scores de riesgo, siendo el STS 2,2\% y el EuroSCORE II $1,76 \%$.

Se realizó el implante valvular aórtico percutáneo con anes- 


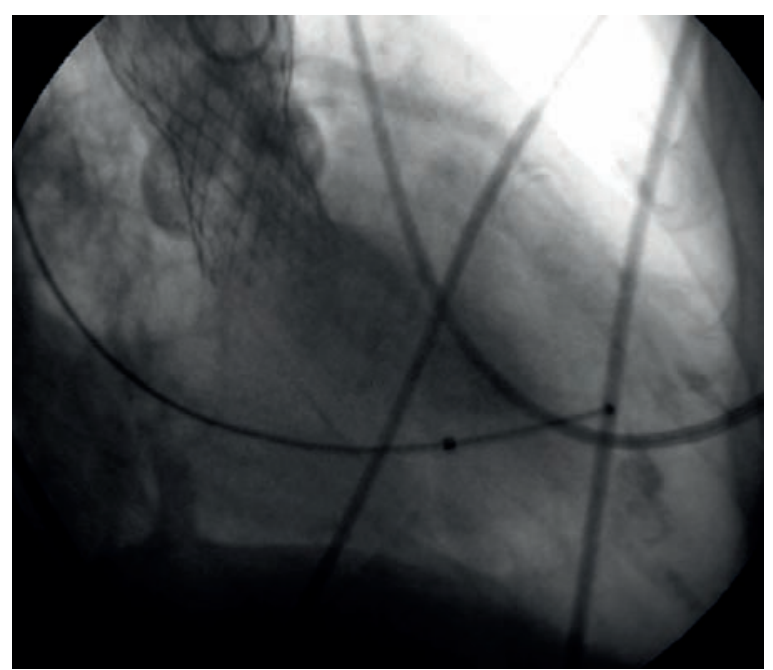

Figura 1. Angiografia aórtica. Proyección oblicua derecha. En ella se evidencia insuficiencia valvular severa.

tesia locorregional. Luego se implantó una prótesis CoreValve $\mathrm{N}^{\circ} 26$ sin complicaciones. En la angiografía control con catéter pig tail posicionado sobre la válvula protésica, se evidenció insuficiencia valvular severa secundaria a leak paravalvular severo, según los criterios de VARC-2 (Figura 1). Se procedió a calcular el IRA, que fue de 27, por lo que se dio por finalizado el procedimiento. Dado que la paciente presentaba en el electrocardiograma basal bloqueo completo de rama derecha, evolucionando con bloqueo auriculoventricular completo, se procedió al implante de un marcapasos bicameral en el mismo acto quirúrgico. Al ingreso a unidad coronaria se realiza ecocardiograma transtorácico donde se observó una insuficiencia valvular leve, con prótesis correctamente implantada y posicionada, normofuncionante (Figura 2). La paciente evolucionó estable y sin complicaciones, por lo que fue dada de alta al tercer día.

\section{DISCUSIÓN}

El reemplazo valvular aórtico percutáneo se ha vuelto un tratamiento alternativo para aquellos pacientes portadores de estenosis aórtica severa sintomática con alto riesgo quirúrgico $^{1,2}$.

Sin embargo, luego del implante, aproximadamente un 70\% desarrolla insuficiencia valvular periprotésica. De estos, un $15 \%-20 \%$ es de grado moderado a severo ${ }^{1-3}$. Las causas de esta complicación son la incompleta y/o mala aposición de la prótesis debido a la excesiva presencia de calcio en el anillo valvular, la altura de implante y la subvaloración de la válvula protésica a implantar ${ }^{2,4}$

Numerosos estudios han demostrado que la presencia de insuficiencia aórtica moderada a severa secundaria a leak paravalvular constituye un factor independiente de mortalidad, tanto a los 30 días como al año luego del implante, por lo que reducir su incidencia o corregirla precozmente, mejora la sobrevida de estos pacientes ${ }^{2,3}$.

Sea cual fuere la causa que generó esta complicación, el tratamiento para resolverla debe realizarse de manera rápida y efectiva. La posdilatación de la válvula con balón, si se evidencia subexpansión de la prótesis; el implante de una segunda válvula o tracción de la ya implantada, si la altura del implante es inadecuada; o la colocación de un dispositivo de cierre si permanece el leak paravalvular luego de la pos-

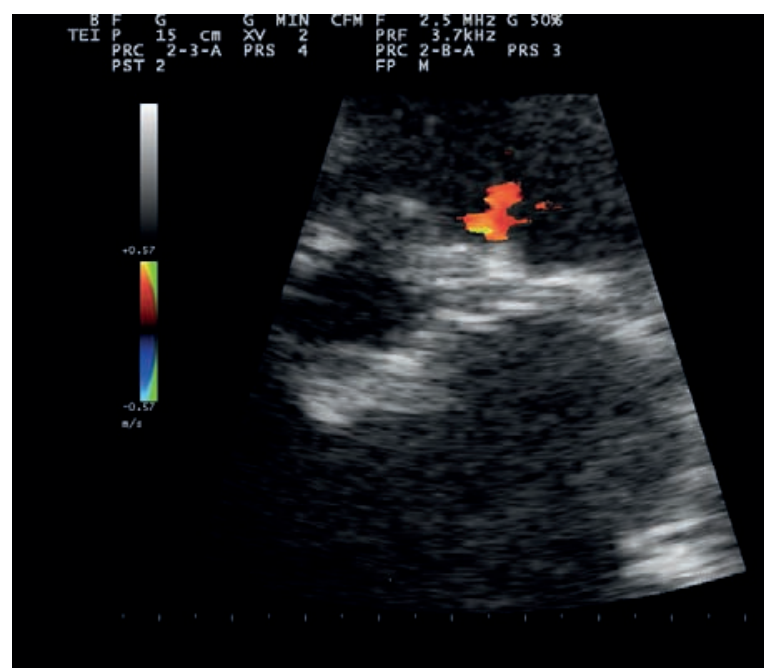

Figura 2. Ecocardiograma transtorácico, vista apical de 5 cámaras centrada en válvula aórtica. Se visualiza insuficiencia valvular leve.

dilatación, constituyen intervenciones correctivas que pueden reducir considerablemente el grado de insuficiencia aórtica $^{1,2}$. Todas estas decisiones terapéuticas deben ser tomadas dentro de la sala de Hemodinamia, por lo que es de gran importancia definir la severidad y el impacto hemodinámico de la insuficiencia aórtica de la manera más rápida y efectiva posible ${ }^{2}$.

Para lograrlo, pueden utilizarse métodos angiográficos, ecocardiográficos y hemodinámicos combinados ${ }^{1,3}$.

El método angiográfico es cualitativo. En la evaluación de la insuficiencia aórtica post-TAVI, la causa más frecuente es el leak paravalvular, que según los criterios de VARC-2 se clasifica en 3 grados según su severidad: leve, moderado o severo. Este método depende de distintos parámetros sujetos a variabilidad: la frecuencia cardíaca, la ubicación del catéter pig tail al momento de la inyección, el flujo y la cantidad de contraste utilizado para realizar la angiografía, y la subjetividad del operador ${ }^{1,3}$

Otro método ampliamente difundido para evaluar el grado de insuficiencia aórtica y la presencia de leak paravalvular es el ecocardiograma transtorácico, pese a que también presenta limitaciones. Los parámetros que mide son semicuantitativos y los cambios hemodinámicos, el calcio presente en la válvula y la prótesis en sí (generando sombra acústica) hacen dificultosa la medición del jet. El ecocardiograma transesofágico presenta mayor precisión, pero requiere sedación y no está exento de riesgos ${ }^{1,2}$.

Dentro de los parámetros hemodinámicos pasibles de evaluación para definir el grado de insuficiencia valvular, se encuentra el índice de regurgitación aórtica (IRA). El mismo es la diferencia entre la presión diastólica aórtica (PDA) y la presión de fin de diástole del ventrículo izquierdo (PFDVI) dividido la presión sistólica aórtica (PSA) multiplicado por $100[$ IRA =(PDA-PFDVI)/PSA x 100]. Este método también depende de la frecuencia cardíaca del paciente, por lo que debe realizarse con una frecuencia cardíaca de entre 60 a 80 latidos por minuto y luego de 10 a 15 minutos posteriores al implante valvular. El valor de corte es 25 , y es inversamente proporcional a la severidad de la insuficiencia aórtica. Un IRA menor a 25 es predictor de insuficiencia valvular moderada a severa con una sensibilidad del $100 \%$ y una especificidad del 68\%, como así también se asocia a un incremento del doble en la mortalidad al año con una sensibilidad del $73 \%$ 
y una especificidad del $66 \%$. Este parámetro permite definir si se debe mejorar el posicionamiento de la válvula luego de realizado el implante ${ }^{1-3}$.

En este caso, pese a que la angiografía posimplante presentaba insuficiencia valvular severa, según los criterios de VARC-2, el IRA fue de 27, por lo que se dio por finalizado el procedimiento, en base al resultado hemodinámico. Al realizarse el ecocardiograma transtorácico control dentro de las 24 hs, pudo observarse una insuficiencia aórtica leve secundaria a leak perivalvular. La paciente fue dada de alta al $3 \mathrm{er}$

\section{BIBLIOGRAFÍA}

1. Werner N, Sinning JM. Aortic regurgitation after transcatheter aortic valve replacement: Nothing to worry about anymore?.Circ J.2014; 78:811-818.

2. Sinning JM, Hammerstingl $C$, Vasa Nicotera $M$, et al. Aortic regurgitation index defines severity of peri-prosthetic regurgitation and predicts outcome in patients after transcatheter aortic valve implantation. J Am Coll Cardiol.2012;59:1134-1141. día del procedimiento, sin complicaciones. En el seguimiento al año, se encontraba asintomática, libre de eventos cardiovasculares mayores, y con un ecocardiograma transtorácico normal, con leak periprotésico leve.

Este caso permite observar la importancia que el "hemodinamista" debe asignarle a la evaluación "hemodinámica" de la patología valvular, conducta actualmente revalorada, con el advenimiento del TAVI, como una práctica habitual en el paciente portador de estenosis aórtica severa sintomática.
3. Vasa Nicotera $M$, Sinning $J M$, Chin $D$, et al. Impact of paravalvular leakage on outcome in patients after transcatheter aortic valve implantation.JACC: cardiovascular interventions.2012;8(5):858-865.

4. Abdel Wahab M, Zahn R, Horack M, et al. Aortic regurgitation after transcatheter aortic valve implantation: incidence and early outcome: results from the German transcatheter aortic valve interventions registry. Heart.2011;97:899-906. 